\title{
Ventilator Associated Pneumonia (VAP) with Multidrug Resistance (MDR) Pathogens in Elderly Patients: Risk Factors and Antibiotic Sensitivity Patterns of MRSA, ESBLs and MBL in Intensive Care Unit
}

\author{
Dr. Rahil Pasha S A, Dr. Priya Bhat, Dr. Abdul Naseer A, Dr. Nikhitha C Ved
}

\begin{abstract}
Ventilator Associated Pneumonia (VAP) is defined as pneumonia that occurs 48 hours or more after endotracheal intubation or tracheostomy, caused by infectious agents not present or incubating at the time mechanical ventilation started1. High mortality and healthcare costs area associated with ventilator-associated pneumonia (VAP) due to Multidrug-Resistant (MDR) Pathogens. The data concerning the link between multidrug-resistance pathogens and outcomes remains controversial2. Therefore, we aimed to identify the relation of risk factors for ventilator-associated pneumonia (VAP) and mortality with the drug resistance profiles of Multidrug-Resistant (MDR) Pathogens with detection of MRSA, ESBLs and MBLs in intensive care unit. This study was conducted in the Department of Microbiology at ESIC MC and PGIMSR, Rajajinagar, Bengaluru from January 2017 to June 2018 . A total of 38 isolates from 35 VAP patients were collected during the study. They were processed following standard laboratory protocol. Antibiogram was done using appropriate antibiotics by Kirby-Bauer disc diffusion method and the occurrence of MRSA, ESBLs and MBLs was seen. Males were most commonly affected, Acinetobacter spp. (40\%), was most common organism isolated followed by Klebsiella pneumoniae (33.33\%). For MDR isolates most sensitive drug was Cefoperazone-sulbactum (25\%), followed by Piperacillin-tazobactam (8.3\%), Piperacillin (8.3\%) and Cefoperazone (8.3\%). Whereas in non-MDR isolates Amikacin (77.7\%) was most sensitive followed by Cefoperazone-sulbactum and Gentamicin (72.2\% each). Most common mechanism of resistance among MDR isolates was found to be Carbapenemase production (53.3\%) 44 by Acinetobacter spp, 2 by Klebsiella pneumoniae, 1 each by Pseudomonas aeruginosa and Escherichia coli\}, followed by AmpC (18.2\%) \{4-Klebsiella pneumoniae\& 2-Escherichia coli\}, and ESBL (3.3\% by Klebsiella pneumoniae). Among Carbapenemase Metallo-betalactamase production was seen in $37.5 \%$ of isolates. Diabetes mellitus (58.33\%) was most common risk factor, followed by smoking (50\%), and alcohol (41.7\%). $88 \%$ of patients had leucocytosis with mean total leucocytosis count (TLC) of 17,348 cells/mm3 and 17\% of patients were anaemic with mean $\mathrm{Hb}$ of $10.02 \mathrm{~g} / \mathrm{dl}$ and $41.7 \%$ of patients had pneumonic changes (consolidation) and 50\% of patients had BL/UL alveolar or interstitial infiltration and 1 patient (8.33\%) had consolidation with CA lung. Periodic analysis of Sputum culture and their antibiotic sensitivity report should be made to identify the changing trends in etiological and sensitivity patterns.
\end{abstract}

Keywords: Ventilator associated pneumonia, Multidrug resistant, Geriatric VAP, Extended Spectrum $\beta$-lactamases, Metallobetalactamases

\section{Introduction}

Ventilator-associated pneumonia (VAP) is one of the most dreaded nosocomial infection and a major threat for the older population, as they have age related immunological changes, chronic cognitive and physical impairment and alter host resistance, and therefore they are highly susceptible to infections and their complications. ${ }^{3}$ Infectious disease account for one third of all deaths in elderly age group. ${ }^{4,5}$ The impact of infectious disease particularly in the ageing population should not be measured only in terms of mortality rate, but also by morbidity and quality of life. ${ }^{6}$ The ageing population has both medical and sociological problems. Ageing in India is exponentially increasing due to the impressive gains that society has made in terms of increased life expectancy including the advances in antibiotic therapy. The elderly population suffers high rates of morbidity and mortality due to infectious diseases. ${ }^{7}$ LRTI (along with pneumonia) a disease of developing countries, have an incidence of about $20 \%-30 \%$ in developing countries like India as compared to $3 \%-4 \%$ in developed countries. ${ }^{8}$ In critically ill patients, the susceptibility of the bacteria isolated in a VAP depends on the duration of stay in the ICU and on mechanical ventilation as well as the previous use of antibiotics. ${ }^{9}$ Acute Lower respiratory tract infections such as pneumonia, acute bronchitis and Acute exacerbations of Chronic Obstructive Pulmonary Disease (COPD) are among the most common reasons to visit a general practitioner (GP), notably among elderly person. ${ }^{10}$ According to the global burden of Disease 2015 study (GBD 2015), COPD and Lower Respiratory Tract Infection represents the $3 \mathrm{rd}$ and 4 th most common cause of death respectively after ischemic heart disease and cerebrovascular disease. $^{11}$

Diagnosing of VAP is difficult as it requires a thorough assessment of clinical findings, radiological findings, and microbiological results. There are no fool proof tools to determine whether the patient has a VAP. When the clinical suspicion of VAP is high, empirical antimicrobial therapy must be initiated promptly because both delayed and inadequate treatments have been associated with increased rate of morbidity and mortality. ${ }^{12}$ In patients with no signs of severe sepsis or septic shock and no organisms present on Gram's staining, antimicrobial therapy can be withheld pending culture results. ${ }^{13,14}$ Nevertheless, one third of the patients with VAP only exhibit clinical criteria of sepsis. ${ }^{4}$ Current guidelines recommend empirical coverage of Gramnegative bacilli (GNB) with a third or fourth generation cephalosporin, Piperacillin-tazobactam or a Carbapenem in 
combination with a fluoroquinolone or an aminoglycoside. ${ }^{15}$ However, the problem arises when a high proportion of the GNB are resistant to these antibiotics. After a period of neglect, this problem is now receiving the deserved attention of the medical community. ${ }^{16}$ The bacteriological profile of the LRTIs are different in different countries, and also vary with time within the same country, the aetiology of respiratory infections play a significant role in the choice of empirical antibiotics, isolation and hospitalization measures. ${ }^{17,18,19}$ The recent advances in medical technologies, usage of mechanical ventilator and other procedures like bronchoscopes, prior antibiotic prescription even before the availability of culture results and frequent admission to hospital lead to the bacterial colonization and infection. ${ }^{20}$ With the emergence of antibiotic resistant bacteria, the role that hospitals play in the development and spread of organisms becomes an important factor for investigation.

\section{Methodology}

This descriptive study was conducted for a period of 1 year from January to December 2018 at a tertiary care hospital, Bangalore, after obtaining due approval from the Institutional ethics committee.

Source of data: Lower respiratory tract samples of geriatric patients like Broncho-alveolar lavage (BAL) and Endo Tracheal Aspirate submitted to diagnostic Microbiology laboratory ESIC MC \& PGIMSR will be included

\section{Inclusion criteria}

1) Lower respiratory tract samples like BAL \& ET Aspirate of patients aged 60 years or above.

\section{Exclusion criteria \\ 1) Patient on chronic suppressive antimicrobial therapy. \\ 2) Patient diagnosed as pulmonary tuberculosis \\ 3) Patient diagnosed as Retro positive.}

Informed consent was obtained from the patients and strict confidentiality about the patient details was maintained.

\section{Laboratory Methodology}

Collection of ET Aspirate2 $2^{21,22}$ :

12 French (Fr) tracheal aspiration probe was introduced through the Endo Tracheal Tube until resistance encountered (level of the carina in the trachea) and retracted approximately $2 \mathrm{~cm}$ to release of the vacuum and probe delicately removed using turning movements and secretions aspirated into sterile collector tube.

Collection of $\mathrm{BAL}^{21,22}$ :

High volume of saline (100 to $300 \mathrm{~mL}$ ) was infused into a lung segment through the bronchoscope to obtain cells and protein of the pulmonary interstitium and alveolar spaces. A deep sampling of desquamated host cells and secretions was collected.

\section{Processing of Samples ${ }^{23}$}

Tracheal aspirate/ BAL - Most purulent portion of tracheal secretion was taken, $0.1 \mathrm{ml}$ sample was diluted in $9.9 \mathrm{ml}$ sterile physiological solution. $0.01 \mathrm{ml}$ was seeded (calibrated loop) on MacConkey agar, blood agar \& chocolate agar and Incubation at $35 \pm 1^{\circ} \mathrm{C}$ for 24 to $48 \mathrm{~h}$, (chocolate agar, in capnofilia $\left(5 \%\right.$ of $\left.\mathrm{CO}_{2}\right)$ at $35 \pm 1^{\circ} \mathrm{C}$ for 24 to $48 \mathrm{~h}$ ). Plates were evaluated for growth at 24 and 48hours. Bacterial isolates grown in culture were identified by means of Gram's staining and biochemical reactions by standard microbiological techniques. Each colony corresponded to $20,000 \mathrm{CFU} / \mathrm{ml}$ and it was considered ETA positive when the count was $\geq 10^{5} \mathrm{CFU} / \mathrm{ml}$.

\section{Antimicrobial Susceptibility Testing ${ }^{24}$ :}

Antibiotic susceptibility tests were done against antibiotics by using Standard Kirby Bauer disk diffusion method in accordance with Clinical and Laboratory Standards Institute (CLSI) criteria. Every batch of Mueller-Hilton agar and antibiotic discs were tested by using following control strains:

ATCC 25922 Escherichia coli,

ATCC 27853 Pseudomonas aeruginosa and

ATCC 25923 Staphylococcus aureus.

\section{Detection of Resistance Mechanisms}

- Multi-drug resistance (MDR)

- Extended Spectrum $\beta$-Lactamase (ESBL) was detected by Phenotypic disc confirmatory test

- AmpC $\beta$-Lactamase was detected by AmpC Disk test

- Carbapenamase and Metallo- $\beta$ Lactase (MBL) was detected by Modified Carba NP test and EDTA synergy test respectively

\section{Tests to detect methicillin resistant Staphylococcus aureus (MRSA)}

\section{Multi-drug resistance (MDR):}

MDR was defined as acquired non-susceptibility to at least one agent in three or more antimicrobial categories. In present study, a Gram negative bacterium was considered MDR when it is resistant to representative drug from these three groups of antibiotics, $\beta$-lactam (ceftazidime), aminoglycoside (gentamicin) and quinolone (ciprofloxacin).

\section{Detection of ESBL by Disk diffusion test (DDT) ${ }^{\mathbf{2 4}}$ :}

Cefotaxime $(30 \mu \mathrm{g})$ or Ceftazidime disks $(30 \mu \mathrm{g})$ with and without clavulanate $(10 \mu \mathrm{g})$ are used. A difference of $\geq 5 \mathrm{~mm}$ between the zone diameters of either of the cephalosporin disks and their respective cephalosporin/clavulanate disk was taken to be phenotypic confirmation of ESBL production.20 The CLSI recommends that the disk tests be performed with confluent growth on Mueller-Hinton agar.

\section{Modified Amp C Disc method ${ }^{24}$ :}

Briefly, 0.5 McFarland suspension of Escherichia coli ATCC 25922 was inoculated on the surface of MHA plate. A $30 \mu \mathrm{g}$ Cefoxitin disk \& a sterile plain disk inoculated with several colonies of the test organism was placed just beside the Cefoxitin disk almost touching it, with inoculated disk face in contact with the agar surface. After overnight incubation at $37^{\circ} \mathrm{C}$, the plates were examined for either an indentation or a flattening of the zone of inhibition, indicating enzymatic inactivation of Cefoxitin (positive result), or absence of a distortion (negative result). 


\section{Carba NP (CNP) test ${ }^{25}$ :}

CNP A solution was prepared by adding phenol red $(0.05 \%)$ and $\mathrm{ZnSO} 4.7 \mathrm{H} 2 \mathrm{O}(0.1 \mathrm{mmol} / \mathrm{L})$ to Clinical Laboratory Reagent Water; $\mathrm{pH}$ was adjusted to $7.8 \pm 0.1$, and the solution was stored at $4{ }^{\circ} \mathrm{C}$ in amber-coloured bottles for up to 15 days. The $\mathrm{B}$ solution was freshly prepared by adding $12 \mathrm{mg} / \mathrm{ml}$ imipenem- cilastatin injectable form (doubling the amount to compensate the cilastatin component; equivalent to $6 \mathrm{mg} / \mathrm{ml}$ of imipenem standard grade powder) to A solution and stored at 40C till use. Two calibrated loops $(10 \mu \mathrm{l})$ of bacterial colony from 18 to $24 \mathrm{~h}$ growth culture from sheep blood agar were re-suspended in $200 \mu$ l of $5 \mathrm{M}$ $\mathrm{NaCl}$ solution and vortexed for 5 seconds. A $100 \mu$ of inoculum was added to two micro centrifuge tubes labelled "a" and "b." Reagents A and B were added to tubes a and b, respectively, incubated at $37^{\circ} \mathrm{C}$ and read at 2 hours. The test was considered positive when tube "a" was red and tube "b" was orange/yellow. In a negative test, both tubes remained red.

\section{Detection of Metallo beta lactamase ${ }^{26}$ :}

Combined disk synergy test (CDST) with $0.5 \mathrm{M}$ ethylene diamine tetra acetic acid Two IPM $(10 \mu \mathrm{g})$ disks were placed $30 \mathrm{~mm}$ apart from center to center on the surface of an agar plate, and $10 \mu 10.5 \mathrm{M}$ EDTA solution was added to one of them to obtain the desired concentration of $750 \mu \mathrm{g}$. If zone of inhibition of IPM-EDTA disk was $\geq 7 \mathrm{~mm}$ more than that of IPM disk alone, it was considered as MBL positive.

\section{Results and Discussion}

A total of 38 organisms (33-gram negative and 5-gram positive) were isolated from 35 patients who developed VAP. Among which 15 isolates were Multi-drug resistant from 12 Patients. Among the 12 patients, 9 (75\%) yielded pure bacterial (mono-microbial) and 3(25\%) yielded mixed infection (two organisms- polymicrobial) \{Figure-1\}.

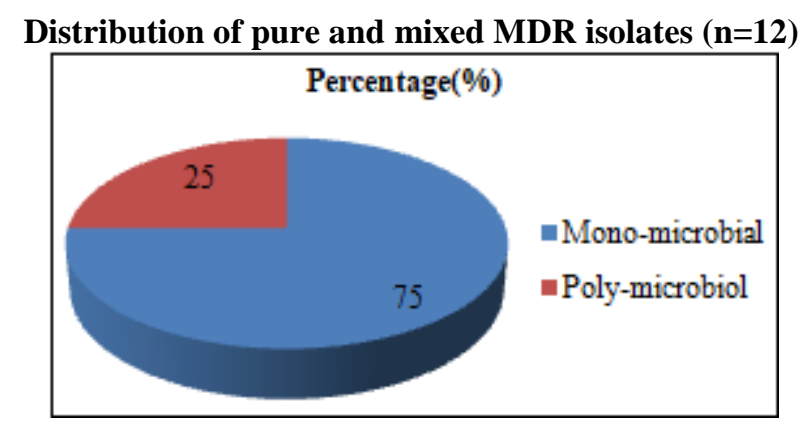

Table 1: Age and Sex wise distribution of MDR Isolates

\begin{tabular}{|c|c|c|c|}
\hline Age group & Female & Male & Total \\
\hline $60-79$ & $4(33.3 \%)$ & $6(50 \%)$ & 10 \\
\hline$\geq 80$ & 0 & $2(16.6 \%)$ & 02 \\
\hline Total & $4(33.3 \%)$ & $8(66.6 \%)$ & 12 \\
\hline
\end{tabular}

Among 12 patients, predominant were males accounting for $66.6 \%$ in which $50 \%$ were between $60-79$ years and $16.6 \%$ were $\geq 80$ years. $33.3 \%$ were females all belonging to 60 79years. (Table -1)
Table 2: Distribution of Poly-microbial isolates

\begin{tabular}{|c|c|c|c|}
\hline Organism & No & Age & Sex \\
\hline Acinetobacter spp + Klebsiella pneumoniae & 1 & 80 & $\mathrm{M}$ \\
\hline Pseudomonas aeruginosa + Escherichia coli & 1 & 72 & $\mathrm{~F}$ \\
\hline Acinetobacter spp + Escherichia coli & 1 & 68 & $\mathrm{~F}$ \\
\hline Total & 3 & & \\
\hline
\end{tabular}

Table 3: Distribution of MDR phenotypes among tracheal

aspirate \& BAL
\begin{tabular}{|c|c|c|}
\hline Organism & MDR & Percentage \% \\
\hline Klebsiella pneumoniae $(\mathrm{n}=7)$ & 5 & 33.33 \\
\hline Acinetobacter spp. $(\mathrm{n}=15)$ & 6 & 40 \\
\hline Pseudomonas aeruginosa $(\mathrm{n}=8)$ & 2 & 13.33 \\
\hline Escherichia coli $(\mathrm{n}=3)$ & 2 & 13.33 \\
\hline Total $(\mathrm{n}=33)$ & 15 & \\
\hline
\end{tabular}

Among Enterobacteriaceae, $33.33 \%$ of Klebsiella pneumoniae and $13.33 \%$ of Escherichia coli were MDR and in Non-Enterobacteriaceae $40 \%$ of Acinetobacter spp., and $13.33 \%$ Pseudomonas aeruginosa were MDR. Overall MDR among Gram negative isolates were $45.5 \%$.

Table 4: Comparison of Antibiotic Resistance among Gram negative isolates $(\mathrm{n}=33)$

\begin{tabular}{|c|c|c|c|c|}
\hline Antibiotic & $\begin{array}{c}\text { MDR } \\
(\mathrm{n}=15)\end{array}$ & $\begin{array}{c}\text { Non-MDR } \\
(\mathrm{n}=18)\end{array}$ & $\%$ \\
\hline Piperacillin & 13 & 86.7 & 13 & 72.2 \\
\hline Ciprofloxacin & 15 & 100 & 9 & 50 \\
\hline Cefoperazone & 14 & 93.3 & 14 & 93.3 \\
\hline Ceftazidime & 15 & 100 & 12 & 80 \\
\hline Piperacillin-tazobactam & 12 & 80 & 7 & 38.9 \\
\hline Cefperazone-sulbactam & 10 & 66.7 & 5 & 27.8 \\
\hline Aztreonem & 14 & 93.3 & 12 & 80 \\
\hline Gentamycin & 15 & 100 & 5 & 27.8 \\
\hline Imipenem & 14 & 93.3 & 7 & 38.9 \\
\hline Meropenem & 12 & 80 & 7 & 38.9 \\
\hline Amikacin & 12 & 80 & 4 & 22.3 \\
\hline
\end{tabular}

For MDR isolates most sensitive drug was Cefoperazonesulbactum (25\%), followed by Piperacillin-tazobactam (8.3\%), Piperacillin (8.3\%) and Cefoperazone (8.3\%). Whereas in non-MDR isolates Amikacin (77.7\%) was most sensitive followed by Cefoperazone-sulbactum and Gentamicin (72.2\% each).

Table 5: Beta lactamase production among MDR Gram negative isolates $(\mathrm{n}=15)$

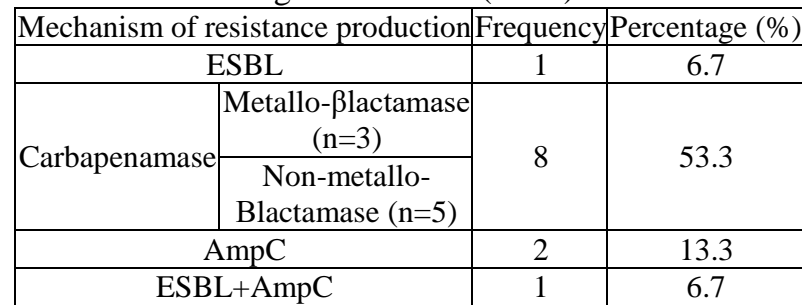

Most common mechanism of resistance among MDR isolates was found to be Carbapenemase production (53.3\%) $\{4$ by Acinetobacter spp, 2 by Klebsiella pneumoniae, 1 each by Pseudomonas aeruginosa and Escherichia coli\}, followed by AmpC (18.2\%) \{4-Klebsiella pneumoniae\& 2Escherichia coli\}, and ESBL $3.3 \%$ by Klebsiella pneumoniae. Among Carbapenemase Metallo-betalactamase production was seen in $37.5 \%$ of isolates. 
Table 6: Correlation with MDR and Carbapenemase among Acinetobacter spp

\begin{tabular}{|c|c|c|c|}
\hline & MDR+ & Non-MDR+ & Total \\
\hline Carbapenamase + & $3(75 \%)$ & 1 & $4(26.67 \%)$ \\
\hline Non carbapenamase + & $1(25 \%)$ & 10 & $11(73.33 \%)$ \\
\hline Total & 4 & 11 & 15 \\
\hline
\end{tabular}

Among the 4 MDR positive Acinetobacter species, 3 isolates were Carbapenamase producers.

Table 7: Correlation with MDR and Carbapenemase among Pseudomonas aeruginosa

\begin{tabular}{|c|c|c|c|}
\hline & $M D R+$ & Non-MDR+ & Total \\
\hline Carbapenamase + & 1 & 1 & $2(25 \%)$ \\
\hline Non carbapenamase + & 1 & 5 & $6(75 \%)$ \\
\hline Total & 2 & 6 & 8 \\
\hline
\end{tabular}

Among the 2 MDR positive Pseudomonas aeruginosa, 1 isolate was Carbapenamase producer.

Table 8: Risk factors associated with MDR Positive VAP infections $(\mathrm{n}=12)$

\begin{tabular}{|c|c|c|}
\hline Risk factor & & Percentage (\%) \\
\hline Diabetic & 7 & 58.33 \\
\hline Smoking & 6 & 50 \\
\hline Alcohol & 5 & 41.67 \\
\hline Previous COPD & 4 & 33.33 \\
\hline Poor oral hygiene & 3 & 25 \\
\hline Cardiac diseases & 2 & 16.67 \\
\hline Malnutrition & 1 & 8.33 \\
\hline Renal disease & 1 & 8.33 \\
\hline Hemiparesis & 1 & 8.33 \\
\hline CA lung & 1 & 8.33 \\
\hline
\end{tabular}

Radiological correlation $(\mathbf{n}=\mathbf{1 2})$

Correlation of chest X-ray was done in all patients, among which $5(41.67 \%)$ patients had pneumonic changes (consolidation) and $6(50 \%)$ patients had $\mathrm{B} / \mathrm{L}$ alveolar or interstitial infiltration and $1(8.33 \%)$ patient had consolidation with CA lung

Table 9: Laboratory correlation $(n=15)$

\begin{tabular}{|c|c|}
\hline Investigation & Percentage $(\%)$ or Mean \\
\hline Anaemia & $17 \%$ \\
\hline Mean Hb & $10.02 \mathrm{~g} / \mathrm{dl}$ \\
\hline Mean TLC & $17348 \mathrm{cells} / \mathrm{mm}^{3}$ \\
\hline Leucocytosis & $88 \%$ \\
\hline
\end{tabular}

\section{Conclusion}

VAP is one of the common infections in the geriatric age group requiring hospitalisation. Age, smoking, and underlying co-morbid conditions especially chronic obstructive pulmonary disease were significantly associated with the development of VAP. The presence of multiple comorbidities, great burden of underlying disease, declining immune status and a different response to treatment with ageing, all increase the susceptibility for Pneumonia in the elderly. We report a high rate of resistance to common antibiotics in present study and Acinetobacter spp to be the most common etiological agent behind the VAP. Furthermore, high level of ESBL and Carbapenamases production is of concern and monitoring of the same is necessary to prevent treatment failure and increased morbidity and mortality among MDR positive VAP cases.
For empirical therapy effective antibiotics found were Imipenem, Amikacin and Meropenem.

Periodic analysis and their antibiotic sensitivity report should be made so that changing trends in the etiological and sensitivity patterns can be identified and therapy adjusted accordingly so that emergence of resistance will be prevented. Strict infection control measures should also be followed to contain hospital acquired infections.

\section{References}

[1] Forbes BA, Sahm DF, Weissfeld AS, editors. Bailey\& Scotte's Diagnostic Microbiology. 12th ed. St. Louis (USA): Mosby Elsevier; 2007. Lower respiratory tract infection; 842-54.

[2] Iginskiene AC, Dambrauskiene A, Rello J and Adukauskiene D. Ventilator-Associated Pneumonia due to Drug-Resistant Acinetobacter baumannii: Risk Factors and Mortality Relation with Resistance Profiles, and Independent Predictors of In-Hospital Mortality. Medicina 2019; 55: 49

[3] C van de Nadort, HM Smeets, J Bont et al. Prognosis of primary care patients aged 80 years and older with lower respiratory tract infection. Br J Gen Pract 2009; 59: 261-65.

[4] Koulenti D, Lisboa T, Brun-Buisson C, Krueger W, Macor A, Sole-Violan J, Diaz E, Topeli A, DeWaele J, Carneiro A, Martin-Loeches I, Armaganidis A, Rello J, EU-VAP/CAP Study Group Spectrum of practice in the diagnosis of nosocomial pneumonia in patients requiring mechanical ventilation in European intensive care units. Crit Care Med. 2009; 37:2360-2368.

[5] Mouton CP, Bazaldua OV, Pierce B, Espino DV. Common infections in older adults. Am Fam Physician 2001; 63(2):257-68.

[6] Gavazzi G, Herrmann F, Krause HK. Aging and infectious diseases in the developing world. Clin Infect Dis 2004; 39:83-91.

[7] Mane AB. Ageing in India: Some social challenges to elderly care. J Gerontol Geriatr Res 2016; 5:136.

[8] Vijay S, Dalela G. Prevalence of LRTI in Presenting with Productive Cough and Their Antibiotic Resistance Pattern. J Clin Diagn Res. 2016; 10(1):09-12.

[9] Trouillet JL, Chastre J, Vuagnat A, Joly-Guillou ML, Combaux D, Dombert MC, Gibert C. Ventilatorassociated pneumonia caused by potentially drugresistant bacteria. Am J Respir Crit Care Med. 1998; 157:531-539. doi: 10.1164/ajrccm.157.2.9705064.

[10] Bont J, Hak E, Hoes A W, Schipper M, Schellevis F G et al. A prediction rule for elderly primary-care patients with lower respiratory tract infections. Eur Respir J. 2007; 29: 969-75.

[11]GBD 2015 Mortality and Causes of Death Collaborators. Global, regional, and national life expectancy, all-cause mortality, and cause of death, 1980-2015: A systematic analysis for the global burden of disease study 2015. Lancet. 2016; 388:1459-544.

[12] Chastre J, Fagon JY. Ventilator-associated pneumonia. Am J Respir Crit Care Med. 2002; 165:867-903.

[13] Canadian Critical Care Trials Group A randomized trial of diagnostic techniques for ventilator-associated pneumonia. N Engl J Med. 2006; 355:2619-2630. 
[14] Chastre J. Conference summary: ventilator-associated pneumonia. Respir Care. 2005; 50:975-983.

[15] American Thoracic Society Guidelines for the management of adults with hospital-acquired, ventilator-associated, and healthcare-associated pneumonia. Am J Respir Crit Care Med. 2005; $171: 388-416$.

[16] Gavazzi G, Herrmann F, Krause HK. Aging and infectious diseases in the developing world. Clin Infect Dis 2004; 39:83-91.

[17] Goel N, Chaudhary U, Aggarwal R, Bala K. Antibiotic sensitivity pattern of gram negative bacilli isolated from the lower respiratory tract of ventilated patients in the intensive care unit. Indian J Crit Care Med. 2009; 13(3):148-51.

[18] Gagneja D, Goel N, Aggarwal R, Chaudhary U. Changing trend of antimicrobial resistance among gram negative bacilli isolated from lower respiratory tract of ICU patients: A 5-year study. Indian J Crit Care Med. 2011; 15(3):164-67.

[19] Kumari HBV, Nagarathna S, Chandramuki A. Antimicrobial resistance pattern among aerobic gramnegative bacilli of lower respiratory tract specimens of intensive care unit patients in a neurocentre. Indian journal of chest diseases and allied sciences. 2007; 49(1):19.

[20] Jackson ML, Neuzil KM, Thompson WW. The burden of community-acquired pneumonia in seniors: results of a population-based study. Clin Infect Dis 2004:39(11):1642-50.

[21] Pereira FO, Menis FA, da Silva BL, Evandro W, Pereira CNC, Alessandro RM.Collection of tracheal aspirate: safety and microbiological concordance between two techniques. Rev. esc. enferm. 2014; 48(4):618-24.

[22] Koenig SM and Truwit JD. Ventilator-Associated Pneumonia: Diagnosis, Treatment, and Prevention. Clin Microbiol Rev. 2006. 19(4):637-57.

[23] J G Collee, W Marr. Specimen collection, culture containers, and media. In: Mackie and McCartney Practical Medical Microbiology. 14th edition. JG Collee, AG Fraser, 113 BP Marmion, A Simmons, editors. Churchill livingstone, Gurgaon Haryana;2014:95-100

[24]Clinical and Laboratory Standards Institute (CLSI). M100-S24. Performance standards for antimicrobial susceptibility testing; 24th informational supplement: Wayne, PA: CLSI; 2014.

[25] Rudresh SM, Ravi GS, Sunitha L, Hajira SN, Kalaiarasan E, Harish BN. Simple, rapid, and costeffective modified Carba NP test for carbapenemase detection among Gram negative bacteria. J Lab Physicians 2017; 9:303-07.

[26] Panchal CA, Oza SS, Mehta SJ. Comparison of four phenotypic methods for detection of metallo- $\beta$ lactamase-producing Gram-negative bacteria in rural teaching hospital. J Lab Physicians 2017; 9:81-83. 